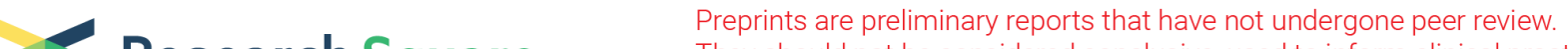 Research Square They should not be considered conclusive, used to inform clinical practice, or referenced by the media as validated information.
}

\section{The Correlation of Internet Addiction and Interpersonal Relationship: A Protocol for a Systematic Review and Meta-Analysis}

Qinghong Hao ( $\sim$ Qinghonghao@hotmail.com )

Chengdu University of Traditional Chinese Medicine https://orcid.org/0000-0001-6163-0960

\section{Wei Peng}

Chengdu University of Traditional Chinese Medicine

Jun Wang

Chengdu University of Traditional Chinese Medicine

Yang Tu

Chengdu University of Traditional Chinese Medicine

Mimi Qiu

Chengdu University of Traditional Chinese Medicine

Yalin Chen

Chengdu University of Traditional Chinese Medicine

Yang Wang

Chengdu University of Traditional Chinese Medicine

Tianmin Zhu

Chengdu University of Traditional Chinese Medicine

\section{Protocol}

Keywords: internet addiction, interpersonal relationship, correlation, adolescents, meta-analysis

Posted Date: August 19th, 2020

DOI: https://doi.org/10.21203/rs.3.rs-60489/v1

License: (1) (1) This work is licensed under a Creative Commons Attribution 4.0 International License.

Read Full License 


\section{Abstract}

Background: Internet addiction has become a very serious social phenomenon. With the popularity of the internet, more and more people are addicted to the virtual network and ignore the real interpersonal communication. This has a lot of adverse effects on the healthy growth of teenagers. Thus, this systematic review and meta-analysis will access the problems existing in the interpersonal relationship of internet addiction and clarify the correlation between internet addiction and interpersonal disturbance.

Method: We will search the following databases: PubMed, Embase, Medline, Web of Science, China National Knowledge Infrastructure (CNKI), Chinese Biomedical Literature Database (CBM), Wan Fang and China Science and Technology Journal Database (VIP). All observational studies will be included. Study quality will be assessed by the Newcastle-Ottawa Scale. We will use Review Manager 5.3 software for bias risk assessment and data synthesis. We will assess the between-study heterogeneity using $\mathrm{I}^{2}$ statistics.

Discussion: We will synthesize the existing research to evaluate the correlation between internet addiction and interpersonal relationship of adolescents. This review will provide new ideas for the further intervention of Internet addiction to some extent.

Systematic review registration: PROSPERO International prospective register of systematic reviews: CRD42020177294

\section{Background}

Internet use has grown substantially in recent decades. According to the 44th China Internet Development Statistics report, the internet penetration rate has exceeded $60 \%$. As of June 2019 , the number of internet users in China reached 854 million, including 847 million mobile internet users [1]. Most studies indicate that the majority of internet users are teenagers, although the internet users are very extensive [2-4]. Based on this, it is extremely important to understand the potential risks associated with Internet use.

Internet addiction (IA) refers to the phenomenon that the internet behavior impulse is out of control under the action of non-addictive substances, and internet users' excessive use of the internet leads to obvious damage to their psychological and social functions [5]. The internet has become a part of people's daily life. Moreover, the problem of internet addiction has aroused widespread concern in the society, and it has been introduced as a risk factor affecting the physical and mental health of teenagers [6]. There are many studies have shown that IA has caused great harm to teenagers' sleep quality [7], mental state $[4,8,9]$, etc.

Interpersonal relationship is a term in the study of social psychology. So far, there is not a particularly fixed definition of interpersonal relationship in academic circles [10]. Generally speaking, interpersonal relationship in a broad sense refers to all kinds of relationships between people, and in a narrow sense, it refers to a direct psychological relationship established by people in the process of communication and 
interaction. Adolescence is the peak period of teenagers' growth and development. During this period, most teenagers produce their own personality characteristics and learn most of the abilities needed for their own survival. Among the many abilities, interpersonal communication ability has a greater impact on teenagers' life[11]. Teenagers' interpersonal skills directly affect their interpersonal relationship, and further affect many aspects of their lives $[3,4,12-16]$. However, new technologies such as the internet are easier to integrate into the lives of teenagers and arouse their great interest [17]. The internet brings them a sense of freshness and satisfaction so that they are increasingly addicted to the internet. This phenomenon not only seriously affects the development of teenagers' physical and mental health, but also brings great trouble to the family and society [18-21]. More importantly, with the promotion and use of the internet, more and more teenagers tend to communicate with the virtual network [22-24] and become more withdrawn and silent in reality $[22,23,25,26]$. Those who have interpersonal barriers in reality are more likely to rely on online friendship and communication to meet their needs.

In recent years, several studies have also shown that there are obvious differences in interpersonal relationship between internet addictions and non-addictions [4, 27-29], which may involve peer relationship, teacher-student relationship, parent-child relationship and so on. School-related stress and poor relationship with teachers, parents, and peers are all risk factors for addictive use [30]. However, successful and effective interpersonal communication is the basis for adolescents to participate in society. If it does not get proper development in adolescence, a series of psychological problems will occur. Studies have proved that IA is an important factor causing interpersonal problems among teenagers. So, it is necessary to study IA from the perspective of teenagers, which can provide guidance for further prevention and reduction of IA. Therefore, we designed this protocol to explore the correlation between internet addiction disorder (IAD) and interpersonal relationship more clearly.

\section{Objectives}

The purpose of this meta-analysis is to further study the problem existing in the interpersonal relationship of internet addictions and clarify the correlation between internet addiction and interpersonal relationship disorders. It also may analyze the differences between different types of interpersonal relationship.

\section{Methods}

We will use the Preferred Reporting Items for Systematic Reviews and Meta-Analyses Protocols (PRISMAP) 2015 [31] statement and guidelines to notify the development of the protocol. The checklist is shown in Table S1.

\section{Study registration}

The protocol of this systematic review (SR) has been registered in PROSPERO (http://www.crd.york.ac.uk/PROSPERO).The registration number is CRD42020177294. Further revisions and additions will be tracked in Prospero. 


\section{Eligibility criteria}

\section{Study designs}

This review covers all studies on the correlation between IA and interpersonal relationship, including all observational studies: cross-sectional studies, case-control studies, and cohort studies. Synthesize the existing research results and make a more clear analysis.

\section{Participants}

All adolescents (ages 13 to 25) who are diagnosed with IAD will be included in this study, regardless of their race and gender, to further study the correlation between IA and interpersonal relationship. The diagnostic criteria are consistent with the fifth edition of the Diagnostic and Statistical Manual of Mental Disorders (DSM-5) [32].

\section{Interventions}

This is an observational study without any intervention is used. This study aims to assess the correlation between IA and interpersonal relationship, in order to further clarify the relationship between them.

\section{Comparators}

Since most of the included studies are observational, there may be no control group. However, if there is a control group, it may be a healthy person without IAD.

\section{Outcome measures}

The primary outcomes are the scale assessment that related to IA and interpersonal relationship, including General situation questionnaire, the Young Internet Addiction Test (YIAT) [33], Chinese Internet Addiction Scale (CIAS) [34], Interpersonal Relationships Integrated Diagnosis Questionnaire (IRIDQ) [35], Quality of life scale for Children adolescents (QLSCA) [36] and so on. Through the comprehensive analysis of the scale score, the relationship between them is discussed. However, the scales used in different studies are different, and we need to consider them comprehensively according to the final included literature.

The secondary outcomes may include parent-child relationship, teacher-student relationship, peer relationship, etc. If possible, we will also summarize and analyze the relationship about different ages, genders, personalities, life experiences and so on.

\section{Exclusion criteria}

The exclusion criteria include:

1) We will be excluded some other types of addiction research and some unrelated research.

2) Some studies with too small sample sizes or missing data should also be excluded. 
3) If duplicate data is involved, it can be recorded only once.

\section{Search strategies}

PubMed, Embase, Medline, Web of Science, China National Knowledge Infrastructure (CNKI), Chinese Biomedical Literature Database (CBM), Wan Fang and China Science and Technology Journal Database (VIP) will be searched for relevant studies in Chinese and English before March 20, 2020. In addition to the electronic databases, we will also search conference papers, dissertations, and reference lists of relevant reviews, so as to ensure the integrity of the inclusion of the literature.

The search strategy for PubMed is as follows:

\begin{tabular}{|ll|}
\hline \#1 & Internet OR online OR social media OR smartphone OR web OR internet game OR Facebook \\
\hline \#2 & addiction OR pathological OR excessive OR disorder OR overuse OR problem \\
\hline \#3 & $\# 1$ AND \#2 \\
\#4 & $\begin{array}{l}\text { Interpersonal Relation OR Relation, Interpersonal OR Relations, Interpersonal OR Social } \\
\text { Interaction OR Interactions, Social OR Social Interactions OR Partner Communication OR } \\
\text { Communication, Partner OR Communications, Partner OR Partner Communications OR peer } \\
\text { relation OR parent-child relation OR teacher-student relation }\end{array}$ \\
\#5 & $\# 3$ AND \#4 \\
\hline
\end{tabular}

\section{Selection of studies}

All retrieved literature will be managed using Endnote $X 9$ and repeated studies will be filtered. The two examiners ( $\mathrm{HQH}$ and $\mathrm{WJ}$ ) will independently screen the study according to the title and abstract based on the pre-set inclusion criteria. The two examiners $(\mathrm{HQH}$ and $\mathrm{WJ})$ will then download the full text of all possible relevant studies and further review the full text independently. Two examiners will cross-examine the included studies. Differences will be resolved through discussion or consensus with third-party reviewers (PW). The program selected for the study will be carried out according to the PRISMA flow chart (see Fig. 1).

\section{Data extractions}

The two researchers $(\mathrm{HQH}$ and $\mathrm{WJ})$ independently extract study data with a pre-defined data extraction form, including: general characteristics (first author, year of publication, language and research country), characteristics of participants (sample size, average age, sex, study population), other characteristics (IAD diagnostic criteria, measurement tools used, interpersonal categories) and outcomes (primary and secondary outcomes). Of course, it also includes more information related to this study.

\section{Risk of bias (quality) assessment}

In this study, the Newcastle-Ottawa Quality Assessment Form (NOS) [37] will be used to assess the quality of the selected study. NOS can be used for case control and cohort studies. Cross-sectional studies were evaluated as case control studies. NOS evaluates three quality parameters, selection, 
comparability, and results. This scale is divided into eight specific projects, which are slightly different in scoring case control and longitudinal study. Each item on the scale is scored with 1 point, except for comparability, which can be adapted to a particular topic of interest, up to a maximum of 2 points. Therefore, the maximum score for each study is 9 . Any study smaller than 5 is identified to be high-risk bias. All stages of the quality assessment process are independently performed by two examiners ( $\mathrm{HQH}$ and $\mathrm{WJ}$ ). The differences are resolved through discussion or consensus with third-party reviewers (TY).

\section{Data analysis}

We will use Review Manager 5.3 (RevMan) for data analysis. A descriptive synthesis will be carried out for all findings in accordance with the inclusion criteria. A quantitative synthesis will also be used if the included studies are homogenous enough.

We will use the $\mathrm{I}^{2}$ index to assess the heterogeneity of effect sizes. $\mathrm{I}^{2}$ index estimates the percentage of variation among effect sizes which can be attributed to heterogeneity. The test of $\mathrm{I}^{2}$ will be used to detect heterogeneity among included studies. An acceptable heterogeneity is defined when $\mathrm{I}^{2} \leq 50$, while substantial heterogeneity is considered when $\mathrm{I}^{2}>50 \%$. We will synthesize the data using a fixed-effects model if the $\mathrm{I}^{2}$ value is $50 \%$ or less, and we will perform meta-analysis. We will use a random-effects model if the $\mathrm{I}^{2}$ value is greater than $50 \%$.

\section{Publication bias}

If the meta-analysis includes 10 or more studies, we will use funnel plots to assess publication bias. When there is no published bias, the figure is symmetrical in the shape of an inverted funnel. When there is publication bias, it will lead to the asymmetry of the funnel diagram and a gap at the bottom. The more obvious its asymmetry is, the more likely it is to have publication bias. If the funnel plots are asymmetrical, we will try to explain the asymmetry of the funnel plot.

\section{Subgroup and sensitivity analyze}

In order to study the potential heterogeneity, it is possible to conduct subgroup analysis and sensitivity analysis.

We will conduct a subgroup analysis according to different age ranges, characteristics of participants, different types of interpersonal relationship, etc. In addition, in order to check the robustness of the combined results, we will conduct a sensitivity analysis to assess the impact of studies with a high risk of deviation.

\section{Ethics and dissemination}

Since this protocol of systematic review and meta-analysis are based on published studies, ethical approval and patient consent are not required.

\section{Discussion}


The interpersonal relationship and IA affect each other. Good interpersonal relationship will inhibit IA, while bad interpersonal relationship will aggravate internet dependence. According to the investigation of Sun Quan [38], the relationship with their parents, classmates and teachers is the order of the degree of interpersonal distress caused by students. The research of Wu Xianhua [39] found that compared with non-internet addicts, internet addicts scored lower in teacher-student relationship, parent-child relationship and peer relationship. It also pointed out that long-term addiction to the internet would make the real interpersonal relationship worse.

To our knowledge, there are many studies on IA and interpersonal relationship, but most of them involve a certain school or a certain type of students. In addition, the choice of research tools is different in different studies, lacking a comprehensive analysis and discussion. Therefore, this meta-analysis will summarize different studies, clarify the interaction between IA and interpersonal relationship. This systematic review(SR) and meta-analysis will not only help internet addicts to build good interpersonal relationship, but also provide new ideas for the intervention of IA to some extent.

\section{Strength and limitations of this study}

This review is based on the previous studies to summarize and analyze, further clarify the correlation between IA and interpersonal relationship. This will provide new ideas for intervention of IA.

However, some studies may choose different scales, the results may have a certain degree of heterogeneity. We will choose subgroup analysis and sensitivity analysis according to different situations.

\section{Abbreviations}

IA

internet addiction; IAD:internet addiction disorder; CINIC:China Internet Network Information Center; SR:systematic review; PRISMA-P:Preferred Reporting Items for Systematic Review and Meta-Analysis Protocols

\section{Declarations}

\section{Acknowledgements}

Not applicable.

\section{Funding}

This research was supported by the Natural Science Foundation of China (81072852 and 81574047$)$, the Key R\&D Project of Sichuan Province (2019YFS0175), the Xinglin Scholar Research Promotion Project of Chengdu University of TCM (XSGG2019007), and the Training Funds of Academic and Technical Leader in Sichuan Province. 
Availability of data and materials

Not applicable.

\section{Authors' contributions}

TZ and WP conceptualized the study protocol and administered the project. All authors (QH, WP, JW, YT, $M Q, Y C, Y W$ and TZ contributed to the development, writing and reviewing of the protocol document. All authors read and approved the final manuscript.

\section{Ethical approval and consent to participate}

Not applicable.

\section{Consent for publication}

Not applicable.

\section{Competing interests}

The authors declare that they have no competing interests.

\section{Author details}

${ }^{1}$ School of Rehabilitation and Health Preservation, Chengdu University of Traditional Chinese Medicine, Chengdu, China

${ }^{2}$ School of Acupuncture and Tuina, Chengdu University of Traditional Chinese Medicine, Chengdu, China

\section{References}

1. CNNIC. The 44th China Internet Network Development Statistics Report. 2019.

2. Sinkkonen HM, Puhakka H, Merilainen M. Internet use and addiction among Finnish adolescents (15-19 years). J Adolesc. 2014;37(2):123-31.

3. Wang P, Zhao M, Wang X, Xie X, Wang Y, Lei L. Peer relationship and adolescent smartphone addiction: The mediating role of self-esteem and the moderating role of the need to belong. $J$ Behav Addict. 2017;6(4):708-17.

4. Seo M, Kang HS, Yom YH. Internet addiction and interpersonal problems in korean adolescents. Comput Inform Nurs. 2009;27(4):226-33.

5. KIMBERLY S.YOUNG PD. Internet addiction:The emergence of a new clinical disorder. 1998.

6. Zhixin Z, Jing G, Hong F, Jingrui X, Xinyi Z, University CN. Study on the effects of Online Learning on the Physical and Mental health of Adolescents. Modern Distance Education 2018(3):59-65. 
7. Chen YL, Gau SS. Sleep problems and internet addiction among children and adolescents: a longitudinal study. J Sleep Res. 2016;25(4):458-65.

8. Pantic I. Online social networking and mental health. Cyberpsychol Behav Soc Netw. 2014;17(10):652-7.

9. Cao F, Su L, Liu T, Gao X. The relationship between impulsivity and Internet addiction in a sample of Chinese adolescents. Eur Psychiatry. 2007;22(7):466-71.

10. Jiangtian W: Analysis of the Adolescent Interpersonal Communication D. Henan University 2013.

11. Rubin KH, Bukowski WM, Parker JG: Peer Interactions, Relationships, and Groups. In: Handbook of Child Psychology. edn.; 2007.

12. Senormancı O, Konkan R, Güçlü O, Senormancı G. Depression, loneliness, anger behaviours and interpersonal relationship styles in male patients admitted to internet addiction outpatient clinic in Turkey. Psychiatr Danub. 2014;26(1):39-45.

13. Ryu H, Lee JY, Choi A, Park S, Kim DJ, Choi JS. The Relationship between Impulsivity and Internet Gaming Disorder in Young Adults: Mediating Effects of Interpersonal Relationships and Depression. Int J Environ Res Public Health 2018, 15(3).

14. Nagase S, Morita N, Ogai Y, Saito T. Evaluation of the relationship between internet addiction and interpersonal relationships in university studentsin Japan. Nihon Arukoru Yakubutsu Igakkai Zasshi. 2016;51(3):184-202.

15. Milani L, Osualdella D, Di Blasio P. Quality of interpersonal relationships and problematic Internet use in adolescence. Cyberpsychol Behav. 2009;12(6):681-4.

16. Liu CY, Kuo FY. A study of Internet addiction through the lens of the interpersonal theory. Cyberpsychol Behav. 2007;10(6):799-804.

17. Baiocco R, Laghi F. Amicizia on-line: disimpegno o stimolazione? 2011.

18. Xin Z, Tianmin Z, Chenchen Z, Lingrui Z, Yu D, Chao W. Influencing factors of adolescents' pathological Internet use. Medical contention. 2018;9(4):30-3.

19. Qian Z, Lei F, Guofu Z, Min L, Jingjing W. Research status of internet addiction in adolescents. Chinese General Practice 2020(13):1687-1694.

20. Qian L. An Analysis of the relationship between Adolescent attachment and Internet overuse. Survey of Education. 2020;9(03):77-9.

21. Cerniglia L, Zoratto F, Cimino S, Laviola G, Ammaniti M, Adriani W. Internet Addiction in adolescence: Neurobiological, psychosocial and clinical issues. Neuroscience \& Biobehavioral Reviews 2016:S0149763416305917.

22. Shapira NA, Goldsmith TD, Keck PE Jr, Khosla UM, McElroy SL. Psychiatric features of individuals with problematic internet use. J Affect Disord. 2000;57(1-3):267-72.

23. Kim K, Ryu E, Chon MY, Yeun EJ, Choi SY, Seo JS, Nam BW. Internet addiction in Korean adolescents and its relation to depression and suicidal ideation: a questionnaire survey. Int J Nurs Stud. 2006;43(2):185-92. 
24. Alavi SS, Maracy MR, Jannatifard F, Eslami M. The effect of psychiatric symptoms on the internet addiction disorder in Isfahan's University students. J Res Med Sci. 2011;16(6):793-800.

25. Alabi OF. A Survey of Facebook Addiction Level among Selected Nigerian University Undergraduates. New Media Mass Commun. 2013;10:70-80.

26. SS A, MR M. F J, M E: The effect of psychiatric symptoms on the internet addiction disorder in Isfahan's University students. Journal of research in medical sciences: the official journal of Isfahan University of Medical Sciences. 2011;16(6):793-800.

27. Xianhua W: Study on Interpersonal Characteristics of Internet Addiction among Adolescents and Its Comprehensive Intervention. D. Huazhong University of Science and Technology; 2013.

28. Ling T: Relationship among interpersonal relationship, Attribution style and Internet Addiction in Secondary vocational students. D. Central China normal University; 2016.

29. Effect of Internet Addiction of College Students on Interpersonal Problems:the Mediating Role of Psy[.... Journal of Guiyang University (Natural Science Edition) 2019, 14(01):17-20.

30. Hui W, Zhou X, Lu C, Jie W, Deng X, Hong L, Scott JG. Problematic Internet Use in High School Students in Guangdong Province, China. Plos One. 2011;6(5):e19660-.

31. Shamseer L, Moher D, Clarke M, Ghersi D, Liberati A, Petticrew M, Shekelle P, Stewart LA. Preferred reporting items for systematic review and meta-analysis protocols (PRISMA-P) 2015: elaboration and explanation. Bmj. 2015;350:g7647.

32. Organization AP: Diagnostic and Statistical Manual of Mental Disorders, 5th ed.. American Psychiatric Association 2013.

33. KS Y: Caught in the net How to recognize the signs of internet addiction-and a winning strategy for recovery. USA: John Wiley $i^{1} / 4 \otimes$ Sons Inc 1998, 89.

34. Shuhui C, Lizhen W, Yiren S, Pinfeng Y. Development of a Chinese Internet Addiction Scale and Its Psychometric Study. Chinese Journal of Psychology. 2003;45(3):279-94.

35. Richang Z. Psychological diagnosis of college students. Jinan: Shandong Education Press 1999:341-399.

36. Hanrong W, Pulin L, Heng M. Norm, Reliability and Validity of Children and Adolescents ' QOL Scale. Chin J School Health 2006, 27(1).

37. Stang A. Critical evaluation of the Newcastle-Ottawa scale for the assessment of the quality of nonrandomized studies in meta-analyses. Eur J Epidemiol. 2010;25(9):603-5.

38. Quan S. Investigation and Counseling Strategies of interpersonal Psychological problems of Secondary Vocational School students. Intelligence 2008:227.

39. Xianhua W, Hanrong W. Interaction effect of association with interpersonal quality and conduct disorder of adolescents with Internet Addiction Disorder. Chinese school health. 2014;35(4):481-3.

\section{Figures}




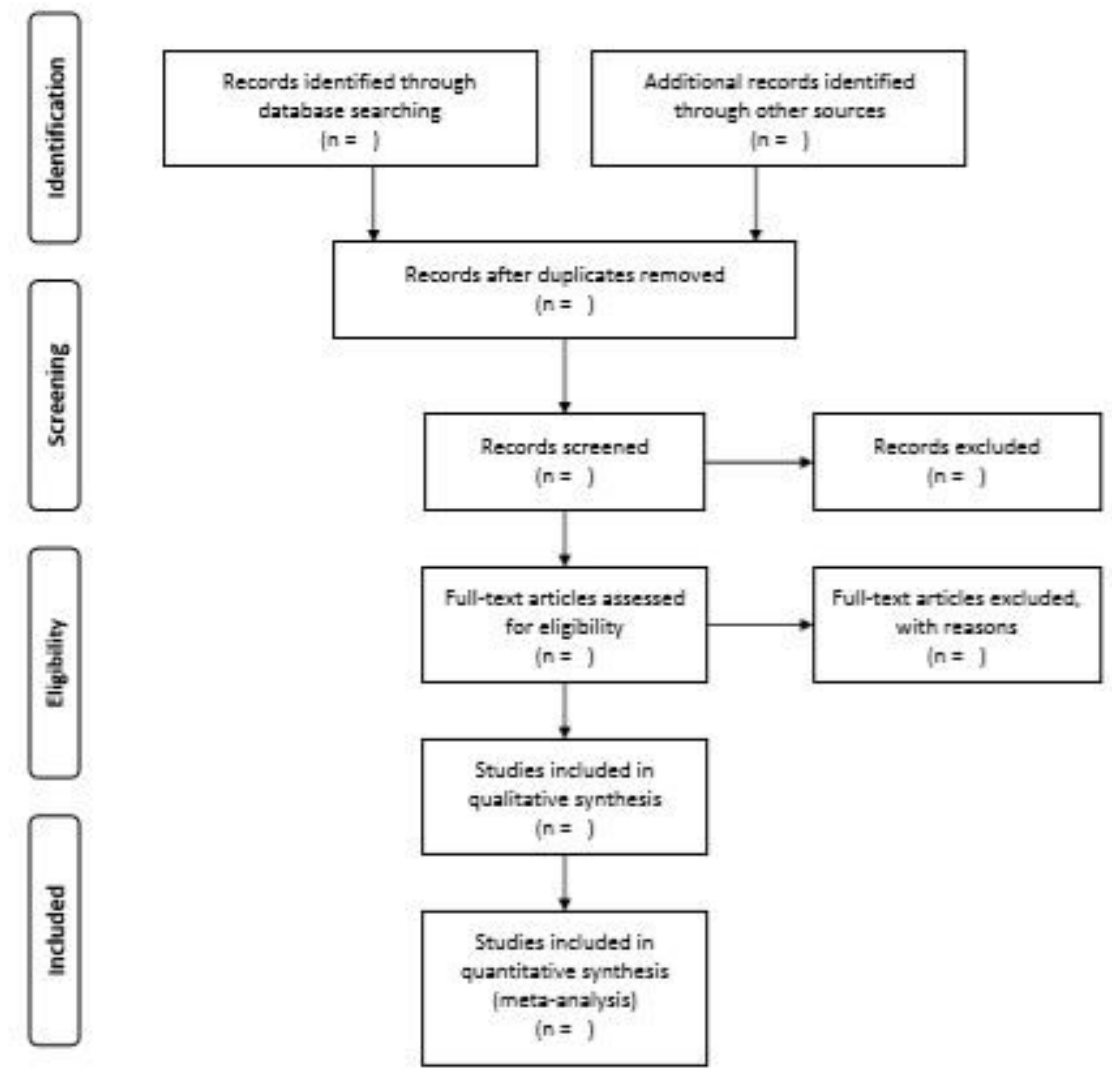

Figure 1

PRISMA 2009 Flow Diagram From: Moher D, Liberati A, Tetzlaff J, Altman DG, The PRISMA Group (2009). Preferred Reporting Items for Systematic Reviews and MetaAnalyses: The PRISMA Statement. PLoS Med 6(6): e1000097. doi:10.1371/journal.pmed1000097

\section{Supplementary Files}

This is a list of supplementary files associated with this preprint. Click to download.

- TableS1PRISMAPchecklist.pdf 